\title{
Detection devices in entanglement-based optical state preparation
}

\author{
Pieter Kok and Samuel L. Braunstein \\ Informatics, University of Wales, Bangor, LL57 1UT, UK
}

\begin{abstract}
We study the use of detection devices in entanglement-based state preparation. In particular we consider optical detection devices such as single-photon sensitivity detectors, single-photon resolution detectors and detector cascades (with an emphasis on the performance of realistic detectors). We develop an extensive theory for the use of these devices. In entanglement-based state preparation we perform measurements on subsystems, and we therefore need precise bounds on the distinguishability of these measurements (this is fundamentally different from, e.g., tomography, where an ensemble of identical states is used to determine probability distributions, etc.). To this end, we introduce the confidence of preparation, which may also be used to quantify the performance of detection devices in entanglement-based preparation. We give a general expression for detector cascades of arbitrary size for the detection up to two photons. We show that, contrary to the general belief, cascading does not give a practical advantage over detectors with single-photon resolution in entanglement-based state preparation.
\end{abstract}

PACS number(s): 42.50.Ar

The accurate creation of quantum states is important to many applications in, for example, quantum computation and information [1,2]. One method of state preparation is to entangle two systems and subsequently perform a so-called conditional measurement on one subsystem: depending on the measurement outcome the undetected subsystem is 'prepared' (collapsed) into a particular predetermined state (see also Rubin [3]). Considerable progress has been made using this method in the creation of optical quantum states 4 - 8 and in the creation of three-photon polarisation entanglement [9]. Optical entanglement sources include, for example, crossKerr media [7] or the mixing of states at beam-splitters [8]. In general, the quality of this entanglement-based state preparation strongly depends on the details of the conditional measurement.

In this paper we study the effect of realistic (photo-) detectors on the state preparation process. To this end we introduce the concept of the confidence of preparation in Sec. If. This measure does not only quantify the 'quality' of the state preparation process, but it also allows us to compare different types of detection devices. In Sec. II we discuss the distinction between single-photon sensitivity and single-photon resolution detectors. The statistics of detector cascading with single-photon sensitivity detectors is studied in Sec. III and Sec. IV makes a numerical comparison between such detector cascades and single-photon resolution detectors.

Let's consider entanglement-based state preparation [3] (not necessarily restricted to quantum optics). We want to prepare a single (pure) state $|\phi\rangle$ by means of some entanglement-based process, and we want the resulting state $\rho$ to be as 'close' to $|\phi\rangle$ as possible. A measure of resemblance between states is given by the fidelity $F$ 10:

$$
F=\operatorname{Tr}[\rho|\phi\rangle\langle\phi|]
$$

The quality of a state preparation process can therefore be measured by the fidelity. When $F=1$, the process gives exactly $|\phi\rangle$ and when $F=0$, the prepared state is orthogonal to $|\phi\rangle$. In practice, the fidelity will not reach these extreme measures, but will lie between 0 and 1 .

For example, if we want to prepare a single-photon state $|1\rangle$ we can use the following process: a parametric down-converter creates a state $|\psi\rangle_{a b}$ on two spatial modes $a$ and $b$ :

$$
|\psi\rangle_{a b} \propto|0\rangle_{a}|0\rangle_{b}+\xi|1\rangle_{a}|1\rangle_{b}+O\left(\xi^{2}\right)
$$

where $|0\rangle$ denotes the vacuum state and we assume $\xi \ll 1$. The higher order terms (included in $O\left(\xi^{2}\right)$ ) consist of states with more than one photon. We now place a photodetector in mode $a$, which 'clicks' when it sees one or more photons (typically, standard detectors can see single photons, but fail to distinguish between one and two photons). Conditioned on such a click, mode $b$ will be in a state

$$
\rho \propto|1\rangle_{b}\langle 1|+O\left(|\xi|^{2}\right)
$$

The fidelity of this process is high: $F=\langle 1|\rho| 1\rangle \simeq 1$, and this is therefore typically a very good single-photon state preparation process (although the situation changes drastically when multiple down-converters are considered [11,12]). Due to the large vacuum contribution, however, the probability of the detector giving a 'click' will be small (of order $O\left(|\xi|^{2}\right)$ ). When the detector does not click, that particular trial is dismissed, hence the conditional character of the detection.

In this example the outcome of the detection is used to either accept or reject a particular run of the state preparation device. However, in general the outcome of the detector can be used to determine a more complicated operation on the remainder of the state preparation process. This is detection plus feed-forward, since the outcome is used further on in the process. An example of this is quantum teleportation, where the outcome of the Bell measurement determines the unitary transformation needed to retrieve the original input state. 
When the measurements in the state preparation process are prone to errors, the state we want to create may not be the state we actually create. This means that errors in the detection devices can lead to reduced fidelities. In this paper we study the effect of detection errors on optical travelling-wave state preparation.

\section{CONFIDENCE}

Consider a preparation device which prepares a state conditioned on a single measurement. For simplicity, we employ two subsystems. One subsystem will be measured, leaving a quantum state in the other. It is clear that prior to the measurement the two systems have to be entangled. Otherwise conditioning on the measurement does not have any effect on the state of the second system.

We can write the total state $|\psi\rangle_{12}$ prior to the measurement in the Schmidt decomposition:

$$
|\psi\rangle_{12}=\sum_{k} c_{k}\left|a_{k}\right\rangle_{1}\left|b_{k}\right\rangle_{2}
$$

with $\left\{\left|a_{k}\right\rangle\right\}_{k}$ and $\left\{\left|b_{k}\right\rangle\right\}_{k}$ orthonormal sets of states for system 1 and 2 respectively. These states correspond to eigenstates of observables $A$ and $B$ with sets of eigenvalues $\left\{a_{k}\right\}_{k}$ and $\left\{b_{k}\right\}_{k}$ respectively. We now measure the observable $A$ in system 1 , yielding an outcome $a_{k}$ (see Fig. 1).

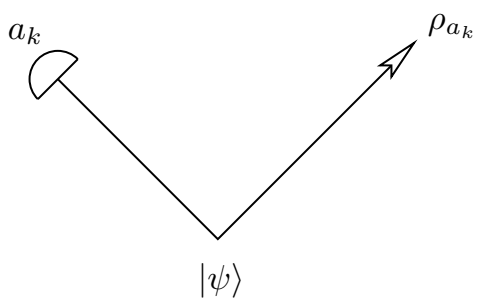

FIG. 1. A schematic representation of state preparation conditioned on a measurement. One branch of the entanglement $|\psi\rangle$ is detected, yielding an eigenvalue $a_{k}$. The other branch is now in a state $\rho_{a_{k}}$.

We can model this measurement using so-called projection operator valued measures, or POVM's for short. For ideal measurements, we can describe the measurement of mode 1 as a projection $P_{k}=\left|a_{k}\right\rangle\left\langle a_{k}\right|$ operating on the state $|\psi\rangle_{12}$. When we trace out the first system the (normalised) state of the second system will be

$$
\rho_{a_{k}}=\frac{\operatorname{Tr}_{1}\left[\left(P_{k} \otimes \mathbb{1}\right)|\psi\rangle_{12}\langle\psi|\right]}{\operatorname{Tr}_{12}\left[\left(P_{k} \otimes \mathbb{1}\right)|\psi\rangle_{12}\langle\psi|\right]}=\left|b_{k}\right\rangle\left\langle b_{k}\right| .
$$

For non-ideal measurements we do not use a projection operator, but rather a projection operator valued measure. In general, a POVM $E_{\nu}$ can be written as 13.14.

$$
E_{\nu}=\sum_{\mu} d_{\mu \nu} \mathcal{P}_{\mu} \geq 0
$$

where the $\mathcal{P}_{\mu}$ 's form a set (possibly over-complete, hence the difference in notation from $P_{k}$ ) of projection operators $\{|\mu\rangle\langle\mu|\}_{\mu}$. We also require a completeness relation

$$
\sum_{\nu} E_{\nu}=\mathbb{1}
$$

As mentioned before, a measurement outcome $a_{k}$ in mode 1 gives rise to an outgoing state $\rho_{a_{k}}$ in mode 2 . We cannot describe a non-ideal measurement with the projection $P_{k}=\left|a_{k}\right\rangle\left\langle a_{k}\right|$. Instead, we have a POVM $E_{k}$ (corresponding to the outcome $a_{k}$ ), which reduces to $P_{k}$ in the case of an ideal measurement. Let $\rho_{12}=|\psi\rangle_{12}\langle\psi|$, the entangled state prior to the measurement. The outgoing state in mode $b$ will then be

$$
\rho_{a_{k}}=\frac{\operatorname{Tr}_{1}\left[\left(E_{k} \otimes \mathbb{1}\right) \rho_{12}\right]}{\operatorname{Tr}\left[\left(E_{k} \otimes \mathbb{1}\right) \rho_{12}\right]},
$$

where the total trace over both systems in the denominator gives the proper normalisation.

If we had an ideal detector (corresponding to $E_{k}=$ $\left.\left|a_{k}\right\rangle\left\langle a_{k}\right|\right)$, the outgoing state would be $\rho_{a_{k}}=\left|b_{k}\right\rangle\left\langle b_{k}\right|$. However, with the general POVM $E_{k}$, this will not be the case. The resulting state will be different. In order to quantify the reliability of a state preparation process we introduce the confidence of a process.

Definition: The confidence in the preparation of a particular state is given by the fidelity of the preparation process.

That means that using Eqs. (14) and (8) the confidence $C$ is given by

$$
C=\frac{\operatorname{Tr}\left[\left(E_{k} \otimes\left|b_{k}\right\rangle\left\langle b_{k}\right|\right) \rho_{12}\right]}{\operatorname{Tr}\left[\left(E_{k} \otimes \mathbb{1}\right) \rho_{12}\right]}=\frac{\left|c_{k}\right|^{2}\left\langle a_{k}\left|E_{k}\right| a_{k}\right\rangle}{\sum_{l}\left|c_{l}\right|^{2}\left\langle a_{l}\left|E_{k}\right| a_{l}\right\rangle},
$$

where the $\left|c_{l}\right|^{2}$ are the diagonal elements of the density matrix. In the context of measurement and state identification, the fidelity is a widely used and well-studied concept 15,16]. Since the confidence is defined as the fidelity of the preparation process, these results also apply here.

We prefer the term confidence in this context, because it is reminiscent of the confidence in statistics [17]. Statistical confidence denotes the probability that the value of a quantity lies within a fixed interval around the observed mean value. In this paper, we extend this meaning to the quantum mechanical case. It is the probability that the prepared state passes a projective test for the expected state in a single-shot experiment.

The confidence $C$ in Eq. (9) can be interpreted as the probability of obtaining outcome $a_{k}$ from the 'branch' containing $\left|a_{k}\right\rangle$ in Eq. (1) divided by the unconditional probability of obtaining outcome $a_{k}$. We will also call this the 'confidence of state preparation'. This interpretation suggests that there does not need to be a second 
system to give the idea of confidence meaning. Suppose, for instance, that we have an 'electron factory' which produces electrons with random spin. A Stern-Gerlach apparatus in the path of such an electron will make a spin measurement along a certain direction $\mathbf{r}$. Suppose we find that the electron has spin 'up' along $\mathbf{r}$. Before this measurement the electron was in a state of random spin $\left(\rho_{\text {in }}=\frac{1}{2}|\uparrow\rangle\left\langle\uparrow\left|+\frac{1}{2}\right| \downarrow\right\rangle\langle\downarrow|\right)$, and after the measurement the electron is in the 'spin up' state $\left(\rho_{\text {out }}=|\uparrow\rangle\langle\uparrow|\right)$. The state of the electron has collapsed into the 'spin up' state. We will now investigate how we can define the confidence of the detection of a single system.

Formally, we can model state collapse by means of the super-operator $\hat{\mathcal{F}}_{a_{k}}$, where $a_{k}$ is again the outcome of the measurement of observable $A$ ('spin up' in the above example). In general, a super-operator yields a (nonnormalised) mapping $\rho \rightarrow \hat{\mathcal{F}}_{\mu}(\rho)$ (see Fuchs and Peres [18,19 and references therein). When the eigenstate corresponding to $a_{k}$ is given by $\left|a_{k}\right\rangle$, we can define the confidence of this measurement as

$$
C_{\mathrm{m}}=\frac{\left\langle a_{k}\left|\hat{\mathcal{F}}_{a_{k}}(\rho)\right| a_{k}\right\rangle}{\operatorname{Tr}\left[\hat{\mathcal{F}}_{a_{k}}(\rho)\right]}=\frac{\operatorname{Tr}\left[\hat{\mathcal{F}}_{a_{k}}(\rho)\left|a_{k}\right\rangle\left\langle a_{k}\right|\right]}{\operatorname{Tr}\left[\hat{\mathcal{F}}_{a_{k}}(\rho)\right]},
$$

with $\operatorname{Tr}\left[\hat{\mathcal{F}}_{a_{k}}(\rho)\right]$ the proper normalisation. However, this expression depends strongly on the details of the family of operators $\mathcal{A}_{\mu \nu}$. This is a more complicated generalisation than the POVM's $E_{k}$. The confidence of state preparation, on the other hand, is a function of the POVM $E_{k}$. Furthermore, $C_{\mathrm{m}}$ will in general not be equal to the confidence of state preparation derived in Eq. (9).

In conclusion, there are two distinct versions of the confidence: the confidence of measurement and the confidence of state preparation. Later in this paper we will use the concept of the confidence to make a quantitative comparison between different detection devices. This suggests that we need to calculate the confidence of measurement with all its difficulties. One way to circumvent this problem is to calculate the the confidence of state preparation using a fixed state. Instead of concentrating on the state preparation process we now choose a standard input state and calculate the confidence for different types of measurement devices. One such choice might be the maximally entangled state

$$
|\Psi\rangle_{12}=\frac{1}{\sqrt{N}} \sum_{k=0}^{N-1}\left|a_{k}, a_{k}\right\rangle .
$$

When $N \rightarrow \infty$, this is perhaps not the ideal choice and another state may be preferred. For any choice, the confidence offers a quantitative measure of performance for different types of measurement devices.

\section{OPTICAL DETECTION DEVICES}

Having set the stage for state preparation conditioned on measurement outcomes, we will now restrict the remainder of this paper to optical implementations. Let's consider the measurement of optical Fock states using photo-detectors. In order to classify different types of detectors we use the following terminology: a detector is said to have a single-photon sensitivity when it is sensitive enough to detect a single-photon wave-packet. This is the case with, for example, the avalanche photo-detector. When a detector can distinguish between $n$ - and $(n+1)$ photon wave-packets, it is said to have a single-photon resolution.

Real detectors have a variety of characteristics. Most common detectors do not have single-photon resolution, although they can distinguish between a few and many photons. When small photon numbers are detected, however, these are single-photon sensitivity detectors to a good approximation. There are also single-photon resolution detectors [20,21]. Currently, these detectors require demanding operating conditions. At this point we note that here, we only consider the detection of single modes. In practice, however, detectors are multi-mode detectors. Since we are dealing with direct detection, these other modes only contribute to the background noise, with the quantum efficiency to the single mode being the key parameter.

When we need single-photon resolution but do not have the resources to employ single-photon resolution detectors, we can use a so-called detector cascade [22]. In a detector cascade an incoming mode (populated by a number of photons) is split into $N$ output modes with equal amplitude which are all detected with single-photon sensitivity detectors. The idea is to choose the number of output modes large enough, so that the probability that two photons enter the same detector becomes small. In general, an optical setup which transforms $N$ incoming modes into $N$ outgoing modes is called an $N$-port (see Fig. 2] [23. A detector cascade is a symmetric $N$-port with detectors at the outgoing modes and vacuum states in all input modes except the first mode. In the next section we will study the statistics of symmetric $N$-ports, but first we need to elaborate on the types of errors which occur in detectors.

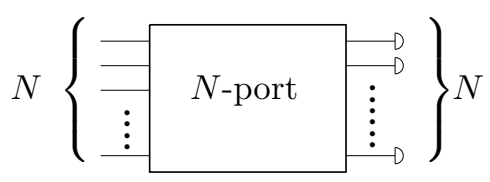

FIG. 2. An $N$-port with unit-efficiency, non-resolving detectors. The $N$ incoming modes are unitarily transformed into $N$ output modes. The $N$-ports considered here consist of mirrors and beam-splitters and do not mix creation operators with annihilation operators.

There are two sources of errors for a detector: it might fail to detect a photon, or it might give a signal although there wasn't actually a photon present. The former may be characterised as a 'detector loss' and the latter as 
a 'dark count'. Here, the emphasis will be on detector losses. In some experiments (like the Innsbruck teleportation experiment [24]) the detectors operate within short gated time intervals. This greatly reduces the effect of dark counts and we will not consider them here.

Detector losses are not so easily dismissed. Every photon entering a detector has a certain probability of triggering it. This probability is called the efficiency of the detector. For the purposes of brevity, when a detector is perfectly efficient, we will call it a unit-efficiency detector. When it has some lower efficiency, we speak of a finite-efficiency detector. Here, we study detector cascading with unit-efficiency detectors, as well as cascading with finite-efficiency detectors [25]. we are interested in the case where cascading distinguishes between photonnumber states $|k\rangle$ and $\left|k^{\prime}\right\rangle$ with $k \simeq k^{\prime}$.

\section{III. $N$-PORTS}

In this section we treat the properties of detector cascades, or symmetric $N$-ports with single-photon sensitivity detectors in the outgoing modes. Symmetric $N$-ports yield a (unitary) transformation $U$ of the spatial field modes $a_{k}$, with $j, k=1, \ldots, N$ :

$$
\hat{b}_{k} \rightarrow \sum_{j=1}^{N} U_{j k} \hat{a}_{j} \quad \text { and } \quad \hat{b}_{k}^{\dagger} \rightarrow \sum_{j=1}^{N} U_{j k}^{*} \hat{a}_{j}^{\dagger},
$$

where the incoming modes of the $N$-port are denoted by $a_{j}$ and the outgoing modes by $b_{j}$. Here, $\hat{a}_{j}^{\dagger}$ and $\hat{a}_{j}$ are the respective creation and annihilation operators of mode $a_{j}$. Similarly for mode $b_{k}$. The unitary matrix $U$ can be chosen to be

$$
U_{j k}=\frac{1}{\sqrt{N}} \exp [2 \pi i(j-1)(k-1) / N]
$$

without loss of generality up to an overall phase-factor. Paul et al. have studied such devices in the context of tomography and homodyne detection [26].

Here, we study $N$-ports in the context of optical state preparation, where only one copy of a state is given, instead of an ensemble. we will use the concept of the confidence, introduced in section $\mathbb{1}$.

\section{A. Statistics of $N$-ports}

Suppose we have a detector cascade, consisting of a symmetric $N$-port with single-photon sensitivity detectors in the outgoing modes. According to Eqs. (12) and (13) incoming photons will be redistributed over the outgoing modes. In this section we study the photon statistics of this device. In particular, we study the case where $k$ photons enter a single input mode of the $N$-port, with vacuum in all other input modes. This device (i.e., the detector cascade) will act as a sub-ideal single-photon resolution detector since there is a probability that some of the photons end up in the same outgoing mode, thus triggering the same detector.

To quantify the single-photon resolution of the cascade we use the confidence given by Eq. (9). Suppose we have two spatially separated entangled modes of the electromagnetic field $a$ and $b$ with number states $|m\rangle$ in $a$ and some other orthogonal states $\left|\phi_{m}\right\rangle$ in $b$ :

$$
|\Psi\rangle=\sum_{m} \gamma_{m}|m\rangle_{a}\left|\phi_{m}\right\rangle_{b}
$$

where the second mode is used only to give the confidence an operational meaning. The POVM governing the detection can be written as $E_{k}=\sum_{m} p_{N}(k \mid m)|m\rangle\langle m|$, since we assume that the photons are not lost in the $N$ port. In this expression $p_{N}(k \mid m)$ is the probability that $m$ incoming photons cause a $k$-fold detector coincidence in the $N$-port cascade. The confidence can then be written as

$$
C=\frac{\left|\gamma_{k}\right|^{2}\left\langle k\left|E_{k}\right| k\right\rangle}{\sum_{l}\left|\gamma_{m}\right|^{2}\left\langle m\left|E_{k}\right| m\right\rangle}=\frac{\left|\gamma_{k}\right|^{2} p_{N}(k \mid k)}{\sum_{m}\left|\gamma_{m}\right|^{2} p_{N}(k \mid m)} .
$$

In order to find the confidence, we therefore first have to calculate the probability distribution $p_{N}$. This will allow us to compare single-photon resolution detectors with various arrangements ( $N$-ports) of single-photon sensitivity detectors.

Suppose $k$ photons enter the first input mode and all other input modes are in the vacuum state. The density matrix of the pure input state $\rho_{0}=|k\rangle\langle k|$ will be transformed according to $\rho=U_{N} \rho_{0} U_{N}^{\dagger}$ with $U_{N}$ the unitary transformation associated with the symmetric $N$-port. Let $\vec{n}$ be the $N$-tuple of the photon number in every outgoing mode: $\vec{n}=\left(n_{1}, n_{2}, \ldots, n_{N}\right)$. The probability of finding $n_{1}$ photons in mode 1 and $n_{2}$ photons in mode 2 , et cetera, is given by $p_{\vec{n}}=\langle\vec{n}|\rho| \vec{n}\rangle$. Using the $N$-port transformation this probability yields

$$
p_{\vec{n}}=\left\langle\vec{n}\left|U_{N} \rho_{0} U_{N}^{\dagger}\right| \vec{n}\right\rangle=\left|\left\langle\vec{n}\left|U_{N}\right| \vec{k}\right\rangle\right|^{2},
$$

where $\vec{k}=(k, 0, \ldots, 0)$, since only the first input mode inhabits photons and the rest are vacuum. From Refs. [27] and 28] we find that this can be rewritten as

$$
p_{\vec{n}}=\frac{\left[H_{\vec{k} \vec{n}}^{R}(0)\right]^{2}}{n_{1} ! \cdots n_{N} ! k !} .
$$

Here, $H_{\vec{k} \vec{n}}^{R}(\vec{x})$ is a so-called multi-dimensional Hermite polynomial (MDHP) [29] and the matrix $R$ is defined as

$$
R \equiv\left(\begin{array}{cc}
0 & -U^{\dagger} \\
-U^{\dagger} & 0
\end{array}\right)
$$

For our present purposes it is convenient to characterise the $N$-port by its transformation of the field modes given 
by Eqs. (12) and (13). we therefore concentrate on $U$ rather than $U_{N}$.

Since there is a one-to-one correspondence between the $N$-port $(U)$ and the matrix $R$, knowledge of $U$ is sufficient to calculate the confidence of a given event using the $N$-port. The MDHP for $N$ input modes with $k$ photons in the first mode and zero in the others (giving an $N$-tuple $\vec{k}$ ) and $N$ output modes $\vec{n}$ is given by

$$
H_{\vec{k} \vec{n}}^{R}(\vec{x})=(-1)^{2 k} e^{\frac{1}{2} \vec{x} R \vec{x}^{T}} \nabla_{\vec{k} \vec{n}}^{2 k} e^{-\frac{1}{2} \vec{x} R \vec{x}^{T}},
$$

where $\vec{x} R \vec{x}^{T}=\sum_{i j} x_{i} R_{i j} x_{j}, \vec{x}=\left(x_{1}, \ldots, x_{2 N}\right)$ and

$$
\nabla_{\vec{k} \vec{n}}^{2 k} \equiv \frac{\partial^{2 k}}{\partial x_{1}^{k} \partial x_{N+1}^{n_{1}} \cdots \partial x_{2 N}^{n_{N}}} .
$$

The number of photons in the input mode is equal to the total number of photons in the output modes. The dimension of $\vec{x}$ obeys $\operatorname{dim} \vec{x}=\operatorname{dim} \vec{k}+\operatorname{dim} \vec{n}=2 N$. For example, for a two-photon input state we have

$$
\left.e^{\frac{1}{2} \vec{x} R \vec{x}^{T}} \frac{\partial^{4}}{\partial x_{1}^{2} \partial x_{l} \partial x_{k}} e^{-\frac{1}{2} \vec{x} R \vec{x}^{T}}\right|_{\vec{x}=0}=2 R_{1 l} R_{1 k} .
$$

There are many different ways in which $k$ incoming photons can trigger a $k$-fold detector coincidence. These different ways correspond to different photon distributions in the outgoing (detected) modes, and are labelled by $\vec{n}_{r}$. The probability that all $k$ photons enter a different detector is found by determining the $p_{\vec{n}_{r}}$ s where every $n_{i}$ in $\vec{n}_{r}$ is at most one. The sum over all these $p_{\vec{n}_{r}}$ 's is equal to the probability $p_{N}(k \mid k)$ of a $k$-fold coincidence in an $N$-port conditioned on $k$ incoming photons:

$$
p_{N}(k \mid k)=\sum_{\vec{n}_{r}} p_{\vec{n}_{r}}=\frac{k !}{N^{k}}\left(\begin{array}{l}
N \\
k
\end{array}\right) .
$$

Finally, in order to find the probability of a $k$-fold detector coincidence conditioned on $m$ photons in the input state (with $m \geq k$ ) we need to sum all probabilities in Eq. (17) with $k$ non-zero entries in the $N$-tuple $\vec{n}$ :

$$
p_{N}(k \mid m)=\sum_{\vec{n} \in \mathcal{S}_{k}} \frac{\left[H_{\vec{m} \vec{n}}^{R}(0)\right]^{2}}{n_{1} ! \cdots n_{N} ! m !},
$$

where $\mathcal{S}_{k}$ is the set of all $\vec{n}$ with exactly $k$ non-zero entries.

\section{B. Realistic $N$-ports}

We now consider a symmetric $N$-port cascade with finite-efficiency single-photon sensitivity detectors. Every one of the $N$ detectors has a certain loss, which means that some photons do not trigger the detector they enter. We can model this situation by putting a beam-splitter with intensity transmission coefficient $\eta^{2}$ in front of the ideal detectors [25]. The reflected photons are sent into the environment and can be associated with the loss. The transmitted photons are detected (see Fig. 3).

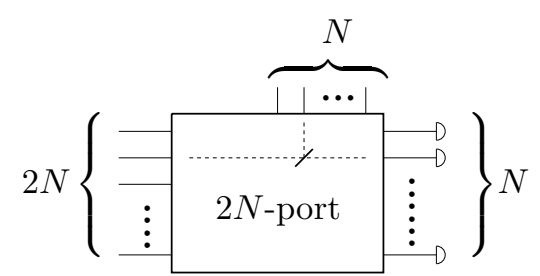

FIG. 3. A $2 N$-port with $N$ modes which are detected with ideal detectors and $N$ undetected modes. These modes are associated with the detector losses.

The implementation of the beam-splitters responsible for the detector losses transform our $N$-port into a $2 N$ port and the unitary transformation $U$ of the field modes in this $N$-port now becomes a $2 N \times 2 N$ unitary matrix $U \rightarrow U \otimes \mathbb{1}_{2}$ (where $\mathbb{1}_{2}$ is the two dimensional unit matrix). Applying a transformation $V_{\eta}$ to implement the beam-splitters with transmission coefficient $\eta^{2}$ will give a new unitary transformation governing the behaviour of the $2 N$-port. Although nothing holds us from considering detectors with different efficiencies, for simplicity we will assume that all detectors have the same efficiency $\eta^{2}$. In terms of the original unitary matrix $U$ from Eq. (13) the new unitary matrix $\widetilde{U}$ becomes

$$
U \rightarrow \widetilde{U}=\left(\begin{array}{cc}
\eta U & \sqrt{1-\eta^{2}} U \\
-\sqrt{1-\eta^{2}} U & \eta U
\end{array}\right)
$$

This changes the matrix $R$ of the MDHP accordingly:

$$
R \rightarrow \widetilde{R}=\left(\begin{array}{cc}
0 & -\widetilde{U}^{\dagger} \\
-\widetilde{U}^{\dagger} & 0
\end{array}\right)
$$

and $\widetilde{R}$ is now a $4 N \times 4 N$ matrix dependent on $\eta$. The probability of finding a $k$-fold detector coincidence in an $\mathrm{N}$-port cascade with finite-efficient detectors then becomes

$$
p_{N}(k \mid m)=\sum_{\vec{n} \in \mathcal{S}_{k}} \frac{\left[H_{\vec{m} \vec{n}}^{\widetilde{R}}(0)\right]^{2}}{n_{1} ! \cdots n_{2 N} ! m !},
$$

where $\mathcal{S}_{k}$ is the set of all $\vec{n}$ with exactly $k$ non-zero entries in the detected modes (note that we still call it an $N$-port although technically it is a $2 N$-port). The confidence of having a total of $k$ photons in a $k$-fold detector coincidence is again given by Eq. (15). The variables of the MDHP will be a $2 N$-tuple $\vec{k}=(k, 0, \ldots 0)$. The output photon number $2 N$-tuple can now be written as $\vec{n}=\left(n_{1}^{d}, n_{2}^{d}, \ldots n_{N}^{d}, n_{1}^{u}, \ldots n_{N}^{u}\right)$, where the superscripts $d$ and $u$ again denote the detected and undetected modes respectively. Furthermore we have $\sum_{i=1}^{N} n_{i}^{d} \equiv N_{d}$ and $\sum_{i=1}^{N} n_{i}^{u} \equiv N_{u}$.

Using Eq. (21) and observing that every detected photon carries a factor $\eta^{2}$ it is quite straightforward to obtain the probability that $k$ photons give a $k$-fold coincidence in an efficient $N$-port cascade:

$$
p_{N}(k \mid k)=\frac{\eta^{2 k} N !}{N^{k}(N-k) !} .
$$




\section{The single-photon resolution of $N$-ports}

Having determined the probability distribution $p_{N}$, we can now calculate the confidence of detector cascading. First of all, in order to obtain a high confidence in the outcome of a detector cascade, the possible number of photons should be much smaller than the number of modes in the cascade: $N \gg k$. In practice there is a limit to the number of detectors we can build a cascade with, so we only look at the lowest order: distinguishing between one and two photons.

we will calculate the confidence of having outgoing state $\left|\phi_{1}\right\rangle$ conditioned a single detector giving a 'click' in the detector cascade when the input state is given by

$$
|\Psi\rangle_{12}=\alpha|0\rangle_{1}\left|\phi_{0}\right\rangle_{2}+\beta|1\rangle_{1}\left|\phi_{1}\right\rangle_{2}+\gamma|2\rangle_{1}\left|\phi_{2}\right\rangle_{2} .
$$

This state corresponds, for example, to the output of a down-converter when we ignore higher-order terms. The confidence is then

$$
C\left(1,|\Psi\rangle_{12}\right)=\frac{|\beta|^{2} p_{N}(1 \mid 1)}{|\alpha|^{2} p_{N}(1 \mid 0)+|\beta|^{2} p_{N}(1 \mid 1)+|\gamma|^{2} p_{N}(1 \mid 2)} .
$$

Eqs. (24) and (20) allow us to calculate the probabilities of a zero-, one- and two-fold detector coincidence conditioned on one or two incoming photons:

$$
\begin{aligned}
& p_{N}(0 \mid 0)=1 \\
& p_{N}(1 \mid 0)=0 \\
& p_{N}(0 \mid 1)=1-\eta^{2} \\
& p_{N}(1 \mid 1)=\eta^{2} \\
& p_{N}(0 \mid 2)=\left(1-\eta^{2}\right)^{2} \\
& p_{N}(1 \mid 2)=\frac{\eta^{4}}{N}+2 \eta^{2}\left(1-\eta^{2}\right) \\
& p_{N}(2 \mid 2)=\frac{N-1}{N} \eta^{4},
\end{aligned}
$$

For example, using these probabilities, together with Eq. (26), gives us an expression for the confidence that a single detector hit was triggered by one photon $(\delta=$ $\left.|\gamma|^{2} /|\beta|^{2}\right)$ :

$$
C=\frac{N}{N+\delta\left[\eta^{2}+2 N\left(1-\eta^{2}\right)\right]},
$$

where, for simplicity, we omitted the functional dependence of $C$ on the incoming state, the size of the cascade and the order of the detector coincidence. This gives a general measure of performance of an cascade of arbitrary size $N$ for the detection of up to two photons. Since the size of the cascade needs to be comfortably larger than the number of detected photons, Eq. (29) will be sufficient for most practical purposes.
A close look at Eq. (28f) shows us that $p_{N}(1 \mid 2)$ includes a term which is independent of the number of modes in the $N$-port cascade. This term takes on a maximum value of $1 / 2$ for $\eta^{2}=\frac{1}{2}$. However, the confidence is a monotonously increasing function of $\eta^{2}$. As expected, for small $\delta$ 's the confidence $C_{N}(1,|\Psi\rangle)$ approaches 1 . Detector cascading thus turns a collection of single-photon sensitivity detectors into a device with some single-photon resolution. In the next section we will give a quantitative estimation of this resolution.

\section{COMPARING DETECTION DEVICES}

Let's return again to the schematic state preparation process depicted in figure 1. There we had two modes, one of which was detected, giving the prepared outgoing state in the other. we argued that different detection devices yield different output states, and the comparison of these states with the ideal case (where we used an ideal detector) led to the introduction of the confidence of a state preparation process. Here, we will use the confidence to make a comparison of different detection devices, rather than output states. This can be done by choosing a fixed entangled input state. The confidence then quantifies the performance of these detection devices.

Consider the state preparation process in the setting of quantum optics. We have two spatial modes of the electro-magnetic field, one of which is detected. In this paper we are mostly interested in states containing a few photons, and the detection devices we consider therefore include single-photon sensitivity detectors, single-photon resolution detectors and detector cascades. As an example, we set the task of distinguishing between one and two photons. Since single-photon sensitivity detectors are not capable of doing this, we will compare the performance of detector cascading with that of a single-photon resolution detector. Let the state prior to the detection be given by

$$
|\Psi\rangle=\frac{1}{\sqrt{3}}\left(|0\rangle\left|\phi_{0}\right\rangle+|1\rangle\left|\phi_{1}\right\rangle+|2\rangle\left|\phi_{2}\right\rangle\right) .
$$

This state is maximally entangled and will serve as our 'benchmark' state. It leads to the choice $\delta=1$ in Eq. (29) in the previous section. Suppose the outgoing state conditioned on a 'one-photon' indication in the detection device is $\rho$. The confidence is then again given by $C=\left\langle\phi_{1}|\rho| \phi_{1}\right\rangle$.

First, consider the single-photon resolution detector described in Refs. 20,21]. This detector can distinguish between one and two photons very well, but it does suffer from detector losses (the efficiency was determined at $88 \%$ ). That means that a two-photon state can be identified as a single-photon state when one photon is lost. The confidence of this detector is therefore not perfect. 
In order to model the finite efficiency of the singlephoton resolution detector we employ the beam-splitter model from section IIIB. We write the input state as

$$
|\Psi\rangle=\frac{1}{\sqrt{3}}\left(|0\rangle\left|\phi_{0}\right\rangle+\hat{a}^{\dagger}|0\rangle\left|\phi_{1}\right\rangle+\frac{\left(\hat{a}^{\dagger}\right)^{2}}{\sqrt{2}}|0\rangle\left|\phi_{2}\right\rangle\right) .
$$

When we make the substitution $\hat{a}^{\dagger} \rightarrow \eta \hat{b}^{\dagger}+\sqrt{1-\eta^{2}} \hat{c}^{\dagger}$ we obtain a state $\rho$. The outgoing density matrix conditioned on a single photon in mode $b$ is then

$$
\begin{aligned}
\rho_{\text {out }} & =\frac{\operatorname{Tr}_{b c}\left[\left(|1\rangle_{b}\langle 1| \otimes \mathbb{1}_{c}\right) \rho\right]}{\operatorname{Tr}\left[\left(|1\rangle_{b}\langle 1| \otimes \mathbb{1}_{c}\right) \rho\right]} \\
& =\frac{\eta^{2}}{4-3 \eta^{2}}\left|\phi_{1}\right\rangle\left\langle\phi_{1}\left|+\frac{4\left(1-\eta^{2}\right)}{4-3 \eta^{2}}\right| \phi_{2}\right\rangle\left\langle\phi_{2}\right| .
\end{aligned}
$$

With $\eta^{2}=0.88$ the confidence of the single-photon resolution detector is easily calculated to be $C=0.65$.

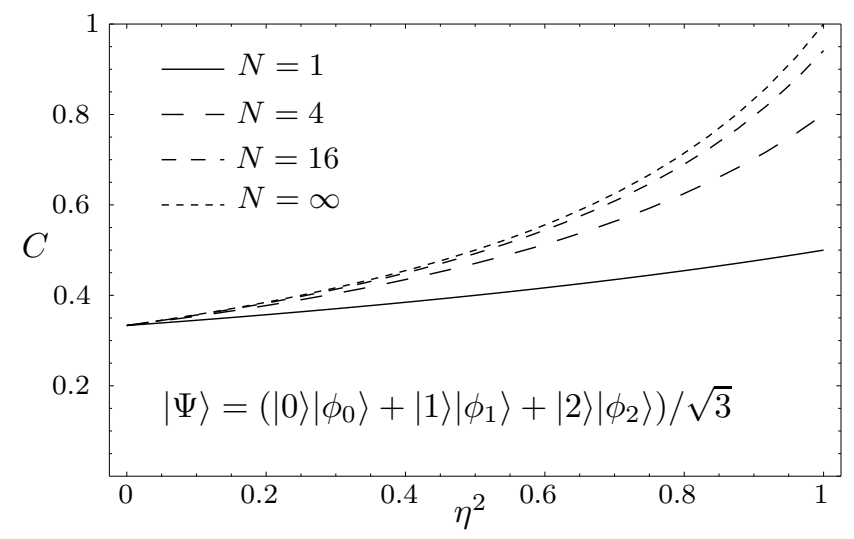

FIG. 4. The single-photon confidence $C$ [Eq. 29)] as a function of the detector efficiency $\eta^{2}$. The solid line corresponds to a single-detector cascade (no cascading: $N=1$ ), the dashed lines correspond to $N=4, N=16$ and $N=\infty$ in ascending order. We consider a maximally entangled input state $|\Psi\rangle=\left(|0\rangle\left|\phi_{0}\right\rangle+|1\rangle\left|\phi_{1}\right\rangle+|2\rangle\left|\phi_{2}\right\rangle\right) / \sqrt{3}$ to serve as a benchmark.

Now we consider a detector cascade with single-photon sensitivity detectors. In Fig. A the confidence of a single-photon detection with $N$-port cascades is depicted. When the cascade consists of four detectors $(N=4)$ it can be easily calculated from Eq. (29) that the detectors need an efficiency of 0.84 to achieve a 0.65 confidence. In the case of infinite cascading $(N=\infty)$ the single-photon confidence of 0.65 is met only if the efficiency is roughly 0.73. This puts a severe practical limit on the efficiency of the single-photon sensitivity detectors in the cascade.

Detector cascading would be practically useful if a reasonably small number of finite-efficiency detectors yields a high confidence. In particular when cascading is viewed as an economical alternative to a detector with singlephoton resolution the number of detectors in the cascade should be small. Additionally, cascading should yield a confidence similar to single-photon resolution detectors. Unfortunately, as a practical application, detector cascading only appears to yield a modest boost in resolution, unless the detectors with single-photon sensitivity have a very high efficiency. In the context of entanglementbased state preparation, real single-photon resolution detectors are therefore superior to detector cascading with currently available detectors, notwithstanding the demanding operating conditions.

\section{CONCLUSIONS}

In this paper we have studied the use of detection devices in entanglement-based travelling-wave state preparation. In particular we considered optical devices such as single-photon sensitivity detectors, single-photon resolution detectors and detector cascades.

Detector cascading has generally been regarded as a good way to enhance single-photon resolution and consequently the fidelity of a state preparation process [22]. However, an extensive theory for the use of these detection devices has not been available so far. The statistics of $N$-ports have been considered in the context of tomography [26], which relies on the availability of a large number of copies of a quantum state. In state preparation, however, we perform measurements on single systems, and we therefore need precise bounds on the distinguishability of these measurements.

To this end, we introduced the confidence of preparation, which can also be used to quantify the (preparation) performance of a (realistic) detection device. We gave an expression for the confidence of a cascade of arbitrary size $N$, conditioned on an input state of up to two photons. We believe that this will be sufficient for most practical purposes. Thus, we compared a single-photon resolution detector with a cascade of single-photon sensitivity detectors and found that cascading does not give a practical advantage over detectors with single-photon resolution for entanglement-based state preparation.

This research was funded in part by EPSRC grant GR/L91344.

[1] See for example chapter 5 of The Physics of Quantum Information, D. Bouwmeester, A. Ekert and A. Zeilinger (Eds.), Springer Verlag (2000).

[2] A. Peres, Quantum Theory: Concepts and Methods, Kluwer Academic Publishers, Dordrecht (1995).

[3] M.H. Rubin, Phys. Rev. A 61, 022311 (2000).

[4] K. Vogel, V.M. Akulin and W.P. Schleich, Phys. Rev. Lett. 71, 1816 (1993).

[5] G. Harel, G. Kurizki, J.K. McIver and E. Coutsias, Phys. Rev. A 53, 4534 (1996).

[6] Special Issue on Quantum State Preparation and Measurement, W.P. Schleich, M.G. Raymer (Eds.), J. Mod. Opt. 44, 11/12 (1997). 
[7] G.M. D'Ariano, L. Maccone, M.G.A. Paris and M.F. Sacchi, Phys. Rev. A 61, 053817 (2000).

[8] M. Dakna, J. Clausen, L. Knöll and D.-G. Welsch, Phys. Rev. A 59, 1658 (1999).

[9] A. Zeilinger, M.A. Horne, H. Weinfurter and M. Zukowski, Phys. Rev. Lett. 78, 3031 (1997).

[10] C.A. Fuchs, PhD thesis, University of New Mexico (1996).

[11] S.L. Braunstein and H.J. Kimble, Nature 394, 840 (1998).

[12] P. Kok and S.L. Braunstein, Phys. Rev. A 61, 42304 (2000).

[13] K. Kraus, States, effects and operations: fundamental notions of quantum theory, Springer Berlin (1983).

[14] C.W. Helstrom, Quantum detection and estimation theory, Academic Press, New York (1976).

[15] S. Massar and S. Popescu, Phys. Rev. Lett. 74, 1259 (1995).

[16] S. Massar and S. Popescu, Phys. Rev. A 61, 062303 (2000).

[17] R. Deutsch, Estimation theory, Prentice-Hall (1965).

[18] C.A. Fuchs and A. Peres, Phys. Rev. A 53, 2038 (1996).
[19] A. Peres, Phys. Rev. A 61, 022116 (2000).

[20] J. Kim, S. Takeuchi, Y. Yamamoto and H. H. Hogue, App. Phys. Lett. 74, 902 (1999).

[21] S. Takeuchi, J. Kim, Y. Yamamoto and H. H. Hogue, App. Phys. Lett. 74, 1063 (1999).

[22] S. Song, C.M. Caves and B. Yurke, Phys. Rev. A 41, R5261 (1990).

[23] M. Reck, A. Zeilinger, H.J. Bernstein and P. Bertani, Phys. Rev. Lett. 73, 58 (1994).

[24] D. Bouwmeester, J.-W. Pan, K. Mattle, M. Eibl, H. Weinfurter and A. Zeilinger, Nature 390, 575 (1997).

[25] H.P. Yuen and J.H. Shapiro, IEEE Trans. Inf. Theory 26, 78 (1980).

[26] H. Paul, P. Törmä, T. Kiss and I. Jex, Phys. Rev. Lett. 76, 2464 (1996).

[27] V.V. Dodonov, O.V. Man'ko and V.I. Man'ko, Phys. Rev. A 50, 813 (1994).

[28] V.V. Dodonov, O.V. Man'ko, V.I. Man'ko and P.G. Polynkin, SPIE 2799, 230 (1996).

[29] V.V. Dodonov, V.I. Man'ko and V.V. Semjonov, Il Nuovo Cimento, 83, 145 (1984). 